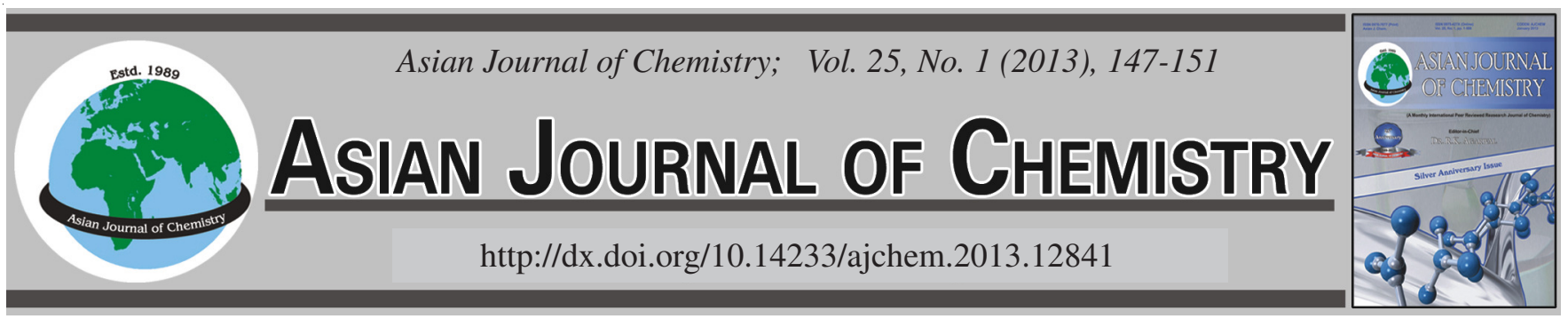

\title{
Identification of Potentially Counterfeit Ingredient in 4-Chlorodehydromethyl Testosterone Tablets by LC-ESI-MS
}

\author{
Shu-Ying HAN ${ }^{1}$, JiAn ZhOU ${ }^{1}$, Hong-Zhen Lian ${ }^{1, *}$ and Li TAN ${ }^{2}$
}

${ }^{1}$ State Key Laboratory of Analytical Chemistry for Life Science, School of Chemistry and Chemical Engineering and Center of Materials Analysis, Nanjing University, 22 Hankou Road, Nanjing 210093, P.R. China

${ }^{2}$ Jiangsu Institute for Drug Control, 6 Beijing West Road, Nanjing 210008, P.R. China

*Corresponding author: Fax: +86 25 83325180; Tel: +86 25 83686075; E-mail: hzlian@ nju.edu.cn

\begin{abstract}
A reversed-phase high performance liquid chromatography-electrospray ionization mass spectrometry method has been developed to identify methyl testosterone from 4-chlorodehydromethyl testosterone in potentially counterfeit steroid products. Counterfeit tablets are suspected to contain not only the declared active pharmaceutical ingredient 4-chlorodehydromethyl testosterone but also the cheaper active ingredient methyl testosterone that is not shown in label. Chromatographic separation was achieved on a Phenomenex Gemini RP I $\mathrm{C}_{18}$ column $(5 \mu \mathrm{m}, 150 \times 4.6 \mathrm{~mm}$ i.d. $)$. Elution was isocratic using a mobile phase consisting of methanol and water $(60: 40$, v/v) at a flow | rate of $1.0 \mathrm{~mL} / \mathrm{min}$. The detection was performed by UV-VIS absorption at wavelength of $254 \mathrm{~nm}$. Excellent linearity between peak area versus standard concentration of methyl testosterone and 4-chlorodehydromethyl testosterone was both obtained in the concentration range of $0.01-0.8 \mathrm{mg} / \mathrm{mL}$. The average recoveries of methyl testosterone and 4-chlorodehydromethyl testosterone in 4-chlorodehydromethyl testosterone tablet were $99.8 \%$ and $100.8 \%$, respectively, with the relative standard deviations of $1.0 \%$ and $1.4 \%$. Moreover, the ESI | mass spectra for these two steroids were presented with the interpretation of fragmentation pattern. Methyl testosterone can be distinguished from 4-chlorodehydromethyl testosterone by its characteristic fragment ions $\mathrm{m} / \mathrm{z} 284.0$ and 270.0 in ESI-MS. The method provides a tool for the control of substandard or counterfeit commercial preparations of steroids.
\end{abstract}

| Key Words: 4-Chlorodehydromethyl testosterone, Counterfeit tablets, Electrospray ionization, Mass spectrometry, Methyl | testosterone, Reversed-phase high performance liquid chromatography.

ᄂ - - - - - - - - - - - - - - - - - - - - - - - - - - - - -

\section{INTRODUCTION}

The counterfeiting of pharmaceuticals has been increasing worldwide, presenting a serious risk for public health. Counterfeit drugs are defined by the world health organization as those that are deliberately and fraudulently mislabeled with respect to identity and/or source. Counterfeiting can apply to both branded and generic products and counterfeit products may include products with correct ingredients, wrong ingredients, without active pharmaceutical ingredients, with insufficient active pharmaceutical ingredients or with fake packaging ${ }^{1}$. Among these the most frequently counterfeiting consists in the absence of active substance, in the presence of a low quantity of active substance, or in the substitution of the declared active ingredient with a cheaper one ${ }^{2}$. Although many previous works had focused on counterfeit drugs used to treat life-threatening conditions, such as antibiotics, antimalarials, antituberculosis and antiretroviral drugs, there have been more and more alarming reports lasting recent years of new and expensive lifestyle counterfeiting medicines, such as hormones, steroids and antihistamines, which have been drawn public attention gradually ${ }^{3}$.

Methyl testosterone (MTS, Fig. 1a) is a synthetic androgenic steroid that has effects of hastening development and maturity of male organ and secondary sexual character, resisting estrogen thus inhibiting endometrium growth and suppressing ovarian and pituitary function. It can promote protein synthesis and sclerotin formation and stimulate bone marrow hematopoiesis for increasing the number of erythrocyte and hemoglobin. Therefore, methyl testosterone has been widely used in clinic for treatment of male hypogonadism, eunuchism, cryptorchidism, menorrhagia, hysteromyoma, endometriosis, senile osteoporosis, childhood aplastic anemia and so on. But, overdosage could lead certain harmful effects on human health ${ }^{4,5}$. 4-Chlorodehydromethyl testosterone (CDM, Fig. 1b) is a derivative of methyl testosterone, which has the similar function as methyl testosterone with a less androgenic but a greater anabolic effect ${ }^{6}$. Methyl testosterone is cheaper than 4-chlorodehydromethyl testosterone, meaning it could be financially beneficial for a counterfeiter to use an 
alternative active ingredient methyl testosterone instead of 4-chlorodehydromethyl testosterone in some steroid preparations. Therefore, a simple and effective method capable of identifying and assaying methyl testosterone and 4-chlorodehydromethyl testosterone is required.
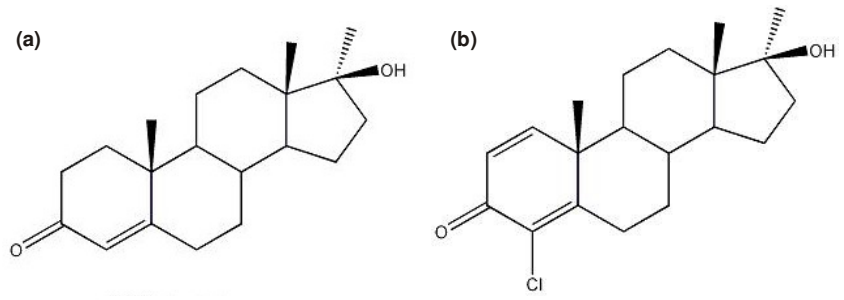

Fig. 1. Structures of methyl testosterone (a) and 4-chlorodehydromethyl testosterone $(b)$

Anticounterfeiting analytical methods range from simple spectrophotometric test ${ }^{7}$ to more sophisticated techniques such as high performance liquid chromatography and liquid chromatography-mass spectrometry approaches ${ }^{8}$. However, neither colourimetric nor HPLC method could be able to identify a substituted wrong active pharmaceutical ingredients. Potentially, this wrong active pharmaceutical ingredients may lead to no active at all. Therefore, identifying a wrong active pharmaceutical ingredients in a drug product presents an interesting analytical challenge. LC-MS is becoming an established technique to help in the pharmaceutical industry ${ }^{9,10}$.

Current analytical methods for methyl testosterone generally rely on spectrophotometry ${ }^{11}$, HPLC $^{12,13}$ and gas chromatography-mass spectrometry ${ }^{14-16}$. Its MS spectrum in electron impact (EI) mode was also given ${ }^{17}$. However, as far as we know the analysis of 4-chlorodehydromethyl testosterone has not been reported in literature yet. In this work, the qualitative electrospray ionization mass spectral information for TMS and 4-chlorodehydromethyl testosterone is studied for the first time. This paper describe a simple LC-ESI-MS protocol for detection of potential counterfeit ingredient methyl testosterone in 4-chlorodehydromethyl testosterone tablet. The method has been successfully applied to the analysis of commercial steroids obtained on the market and has been proved to show great potentials as a screening tool for pharmaceutical counterfeit steroids detection.

\section{EXPERIMENTAL}

Reference substance of methyl testosterone (99\%) was purchased from the National Institute for the Control of Pharmaceutical and Biological Products (Beijing, China). Reference substance of 4-chlorodehydromethyl testosterone (98\%) was provided by Changzhou Huali Chemical Factory (Changzhou, China). Methanol (HPLC grade) was from Merck (Darmstadt, Germany). Wahaha purified water (Wahaha Group Ltd., Hangzhou, China) was used throughout the experiment.

\section{Preparation of standard and sample solutions}

Standard solution: Stock solution of the mixture of methyl testosterone and 4-chlorodehydromethyl testosterone was prepared by accurately weighing about $40 \mathrm{mg}$ reference substances into a 50-mL volumetric flask and then dissolving in methanol. The standard solutions of other mixture were prepared from this stock solution by serial dilution with methanol in the further stages of the experiment.

Sample solution: For method validation of 4-chlorodehydromethyl testosterone five tablets were weighed together. Then these tablets were placed in an agate mortar and grinded into powder sufficiently. About $10.00 \mathrm{mg}$ drug powder was accurately weighed into a $10 \mathrm{~mL}$ volumetric flask and sonicated in an ultrasonic bath for $0.5 \mathrm{~h}$ with about $8 \mathrm{~mL}$ methanol as extraction solvent. Sample solution was completed by adding methanol to the mark and then filtered with $0.45 \mu \mathrm{m}$ filter membrane.

The HPLC system employed was a waters 2695 Alliance separation module (Milford, MA, USA) consisting of a vacuum degasser, a quaternary pump and an auto-sampler, equipped with a waters 996 UV-VIS photodiode-array detector (DAD). The separation was controlled and the chromatograms were recorded by a waters empower chromatography manager system. The separation was performed on a Phenomenex Gemini PP C $_{18}$ column, $5 \mu \mathrm{m}, 150 \times 4.6 \mathrm{~mm}$ i.d. (Guangzhou FLM Scientific Instrument Co. Ltd., Guangzhou, China). The column temperature was maintained at $30^{\circ} \mathrm{C}$. Elution was isocratic using a mixture of methanol and water (60:40, v/v) at a flow rate of $1 \mathrm{~mL} / \mathrm{min}$. The injection volume was $20 \mu \mathrm{L}$ and the detection wavelength was $254 \mathrm{~nm}$.

The LC-MS system comprised of an Agilent technologies 1100 series LC/MSD trap SL system (Palo Alto, CA, USA) with a high pressure double gradient pump, an on-line vacuum degasser, an auto-sampler, a DAD detector and an ion trap mass spectrometer with an electrospray ionization (ESI) interface. All measurements were carried out in positive and negative ion modes, respectively. The LC column was an Agilent Zorbax SB-C ${ }_{18}$ column, $5 \mu \mathrm{m}, 250 \times 4.6 \mathrm{~mm}$ i.d. set at $30^{\circ} \mathrm{C}$. The mobile phase was composed of methanol and water $(75: 25, \mathrm{v} / \mathrm{v})$ at a constant flow rate of $1.0 \mathrm{~mL} / \mathrm{min}$. Injection volume was $10 \mu \mathrm{L}$. Detection was performed on the DAD detector set at $254 \mathrm{~nm}$ and on the mass spectrometer with a full scan mass spectra over the $\mathrm{m} / \mathrm{z}$ range 50-500, a capillary voltage of $3500 \mathrm{~V}$, a collision energy of $0.8 \mathrm{eV}$, a dry temperature of $325^{\circ} \mathrm{C}$, a dry gas (high purity nitrogen) flow rate of $5 \mathrm{~L} / \mathrm{min}$, a nitrogen nebulizer pressure of 5 psi and a dwell time of 300 ms. To measure the second Tandem mass spectrometry (MS2) of methyl testosterone and 4-chlorodehydromethyl testosterone, the quasi-molecular ion of methyl testosterone in negative mode, $[\mathrm{M}-\mathrm{H}]-$ at $\mathrm{m} / \mathrm{z} 301.2$ and that of 4-chlorodehydromethyl testosterone, $[\mathrm{M}-\mathrm{H}]^{-}$at $m / z 333.3$ was selected as the precursor ion in MS/MS experiments, respectively. The collision offset energy were optimized over the range of $0.24-1.6 \mathrm{eV}$ chosen by smart frag model. An Agilent chromatography manager system was used for instrument control and data processing.

\section{RESULTS AND DISCUSSION}

Chromatographic retention: The method allows the separation and simultaneous determination of two different steroids with similar structure. Typical chromatograms of standard solution containing methyl testosterone and 4-chlorodehydromethyl testosterone at $0.1 \mathrm{mg} / \mathrm{mL}$ each and sample solution for method validation under the given chromatographic condition are shown in Fig. 2. A comparison of the retention times in Fig. $2 \mathrm{a}$ and $2 \mathrm{~b}$ preliminarily indicated that 
peaks $1\left(t_{R} 14.9 \mathrm{~min}\right)$ and $2\left(t_{R} 17.3 \mathrm{~min}\right)$ might be methyl testosterone and 4-chlorodehydromethyl testosterone, respectively.
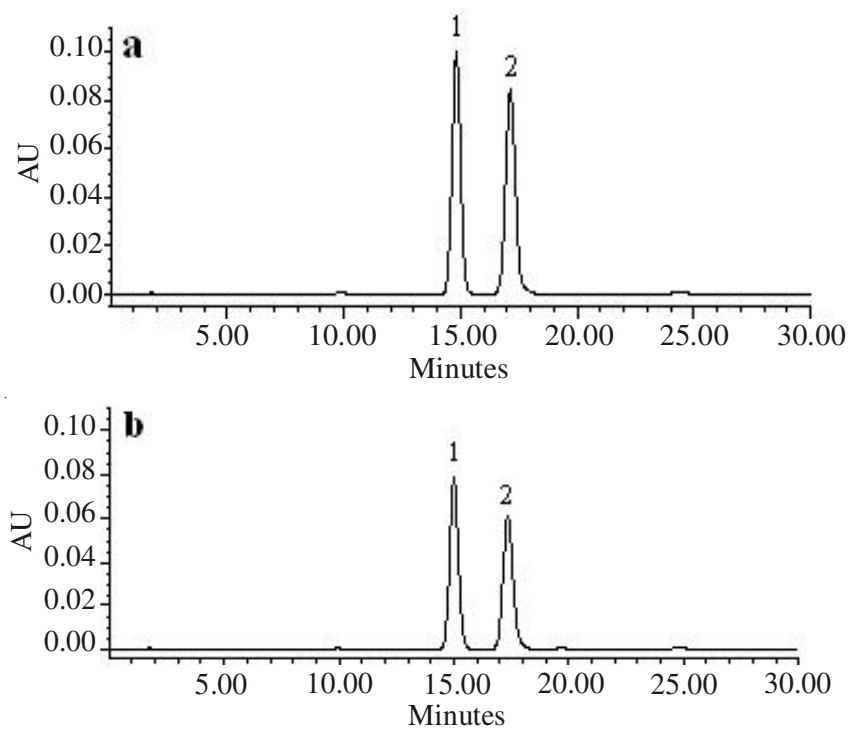

Fig. 2. Chromatograms of standard (a) and sample (b) solutions. Instrumentation: waters 2695 Alliance separation module-996 UVVIS photodiode-array detector (DAD). Column: Phenomenex Gemini $\mathrm{RP}_{18}$ column $(5 \mu \mathrm{m}, 150 \times 4.6 \mathrm{~mm}$ i.d. $)$. Column temperature: $30{ }^{\circ} \mathrm{C}$. Mobile phase: methanol-water $(60: 40, \mathrm{v} / \mathrm{v})$ Flow rate: $1.0 \mathrm{~mL} / \mathrm{min}$. Injection volume: $20 \mu \mathrm{L}$. Detection wavelength: $254 \mathrm{~nm}$. Peaks: 1. methyl testosterone; 2. 4Chlorodehydromethyl testosterone

Ultraviolet spectrum: It can be easily observed from DAD that the UV-VIS spectra corresponding to peaks 1 and 2 in chromatogram of sample solution were in good agreement with those of methyl testosterone and 4-chlorodehydromethyl testosterone in chromatogram of standard solution, respectively, which further confirmed that peaks 1 and 2 were methyl testosterone and 4-chlorodehydromethyl testosterone, respectively (Fig. 3).

Method validation: The system suitability test was performed on both standard solution containing methyl testosterone and 4-chlorodehydromethyl testosterone at $0.1 \mathrm{mg} / \mathrm{mL}$ each and a typical sample solution. The effective theoretical plate numbers ( $\left.n^{\prime}\right)$ of 4-chlorodehydromethyl testosterone and methyl testosterone were listed in Table-1. The resolution between the two peaks met the requirement of baseline separation $\left(R_{S}=2.8\right)$. Moreover, the chromatograms of sample solutions showed that there were no interfering peaks from auxiliary materials in the drug overlapping with those of methyl testosterone and 4-chlorodehydromethyl testosterone.

The reproducibility test was evaluated by injecting the standard solution of the mixture of methyl testosterone and 4chlorodehydromethyl testosterone $(0.1 \mathrm{mg} / \mathrm{mL})$ repeatedly for seven times. The relative standard deviations (RSDs) for peak area of methyl testosterone and 4-chlorodehydromethyl testosterone were $0.6 \%$ and $0.5 \%(n=7)$, respectively.

The linearity between peak area versus standard concentration was acquired in the concentration range of $0.01-0.80$ $\mathrm{mg} / \mathrm{mL}$. The linear equations were $\mathrm{A}_{\mathrm{MTS}}=6202.98+4.48193$ $\mathrm{E} 7 \mathrm{C}_{\mathrm{MTS}}$ for methyl testosterone and $\mathrm{A}_{\mathrm{CDM}}=31956.19+$ 3.33024E7 $\mathrm{C}_{\mathrm{CDM}}$ for 4-chlorodehydromethyl testosterone, respectively, with correlation coefficients (r) of 1.0000 and 0.9998. Limits of detection, with the criterion of a signal-tonoise ratio of $3(\mathrm{~S} / \mathrm{N}=3)$, were $6.25 \times 10^{-5} \mathrm{mg} / \mathrm{mL}$ and $1.25 \times$ $10^{-4} \mathrm{mg} / \mathrm{mL}$ for methyl testosterone and 4-chlorodehydromethyl testosterone, respectively.
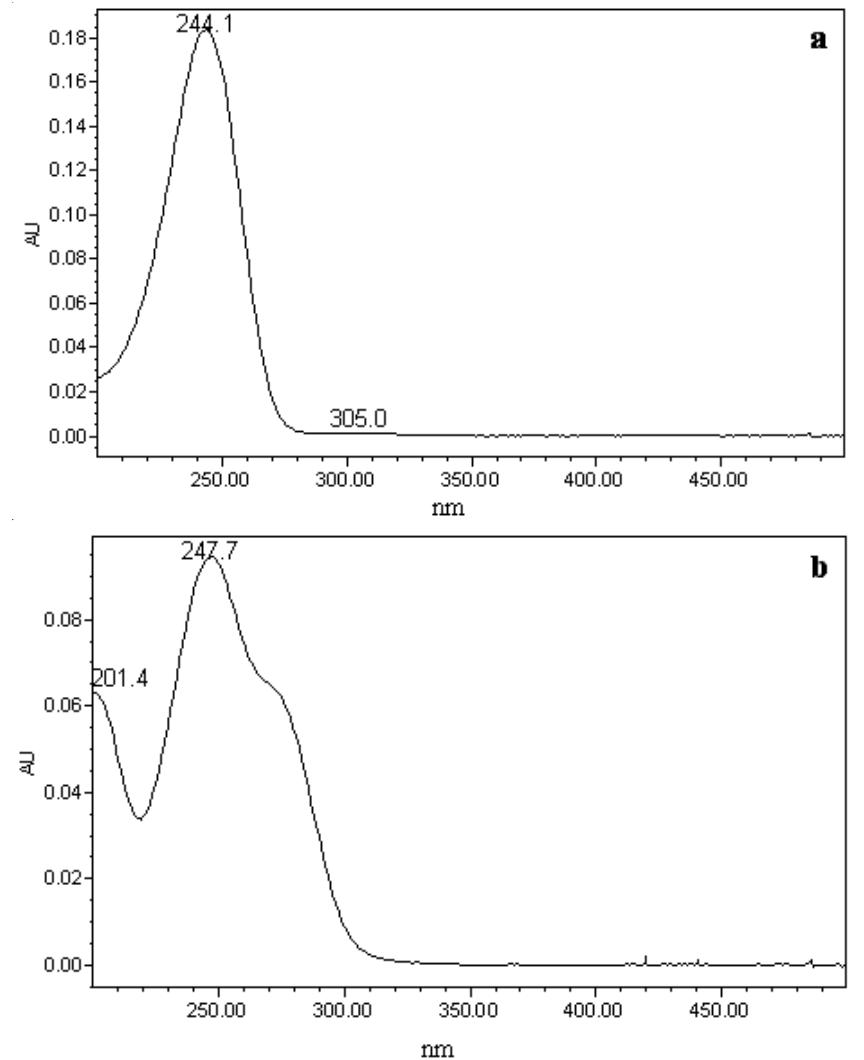

Fig. 3. UV spectra of methyl testosterone (a) and 4-chlorodehydromethyltestosterone (b) from on-line DAD in Fig. 2. Scanning wavelength range: 200-500 $\mathrm{nm}$. Other HPLC conditions were the same as in Fig. 2

\begin{tabular}{lcccccc}
\multicolumn{7}{c}{ TABLE-1 } \\
SYSTEM SUITABILITY & & \\
\hline Solutions & Analyte & $\begin{array}{c}\mathrm{t}_{\mathrm{R}} \\
(\mathrm{min})\end{array}$ & $\mathrm{t}_{0}(\mathrm{~min})^{[\mathrm{a}]}$ & $\begin{array}{c}\mathrm{W}_{1 / 2} \\
(\mathrm{~min})\end{array}$ & $\mathrm{n}^{\prime}$ & $\mathrm{R}_{\mathrm{s}}$ \\
\hline Standard & MTS & 14.91 & 1.75 & 0.41 & 5704 & 2.8 \\
& CDM & 17.33 & 1.75 & 0.46 & 6379 & \\
\hline Sample & MTS & 15.00 & 1.75 & 0.40 & 5926 & 2.8 \\
& CDM & 17.35 & 1.75 & 0.46 & 6452 & \\
\hline${ }^{[\mathrm{a}]}$ Methanol was employed as the hold-time marker & &
\end{tabular}

The stability experiment was performed by analyzing a sample solution for method validation at interval of $0,1,3,7$, 15 and $32 \mathrm{~h}$. It is found that the RSDs of concentration for methyl testosterone and 4-chlorodehydromethyl testosterone were $2 \%$ and $1.6 \%(n=6)$, respectively. The result revealed that methyl testosterone and 4-chlorodehydromethyl testosterone in sample solutions would remain stable at least in $32 \mathrm{~h}$.

The quantitative determination of methyl testosterone and 4-chlorodehydromethyl testosterone in powder of 4chlorodehydromethyl testosterone tablet was carried out on the basis of corresponding standard curves. The contents of the two steroids in drug powder, together with intra- and inter-day precisions, are summarized in Table-2. 
TABLE-2

DETERMINATION OF METHYL TESTOSTERONE AND 4-CHLORODEHYDROMETHYL TESTOSTERONE IN SAMPLE TABLET

\begin{tabular}{cccc|ccc}
\hline \multirow{2}{*}{ Analyte } & Content $(\%)$ & $\begin{array}{c}\text { Average } \\
(\%)\end{array}$ & $\begin{array}{c}\text { Intra-day RSD } \\
(\%)(\mathrm{n}=6)\end{array}$ & Content $(\%)$ & $\begin{array}{c}\text { Average } \\
(\%)\end{array}$ & $\begin{array}{c}\text { Inter-day RSD } \\
(\%)(\mathrm{n}=5)\end{array}$ \\
\hline MTS & $5.86,5.73,5.76,5.80,5.83,5.86$ & 5.81 & 0.9 & $5.81,5.86,5.70,5.81,5.73$ & 5.78 & 1.2 \\
CDM & $7.20,7.03,6.94,7.11,7.17,7.18$ & 7.11 & 1.4 & $7.11,7.14,7.17,7.16,7.17$ & 7.15 & 0.4 \\
\hline
\end{tabular}

TABLE-3

RECOVERIES OF 4-CHLORODEHYDROMETHYL TESTOSTERONE (CDM)

AND METHYL TESTOSTERONE (MTS) IN SAMPLE TABLETS $(\mathrm{n}=3)$

\begin{tabular}{ccccccc}
\hline Analyte & Original $(\mathrm{mg} / \mathrm{mL})$ & Added $(\mathrm{mg} / \mathrm{mL})$ & Found $(\mathrm{mg} / \mathrm{mL})$ & Recovery $(\%)$ & Average recovery $(\%)$ & RSD $(\%)$ \\
\hline \multirow{3}{*}{ MTS } & 0.0585 & 0.0400 & 0.0981 & 99.49 & & \\
& 0.0583 & 0.0800 & 0.1370 & 99.06 & 99.8 & 1.0 \\
& 0.0590 & 0.1600 & 0.2213 & 100.91 & & \\
CDM & 0.0716 & 0.0401 & 0.1050 & 99.86 & & 100.8 \\
& 0.0713 & 0.0802 & 0.1426 & 99.98 & 1.4 \\
\hline
\end{tabular}

The recovery was evaluated by adding known amount of standard solution of the mixture of methyl testosterone and 4chlorodehydromethyl testosterone into sample, which was dealt by the same procedure as the sample solution for method validation mentioned above before assaying. Three levels of addition were performed for the test (Table-3).

\section{Optimization of chromatographic conditions}

Mobile phase: Different methanol proportion in the mobile phase was tested to search for optimum elution condition (Fig. 4). The retention time of methyl testosterone and 4-chlorodehydromethyl testosterone increased rapidly with the decrease of methanol proportion from 80 to $50 \%$. When $60 \%$ methanol was employed, a satisfactory separation was obtained. Therefore, the mixture methanol-water $(60: 40$, v/v) was selected as the mobile phase in present experiments.

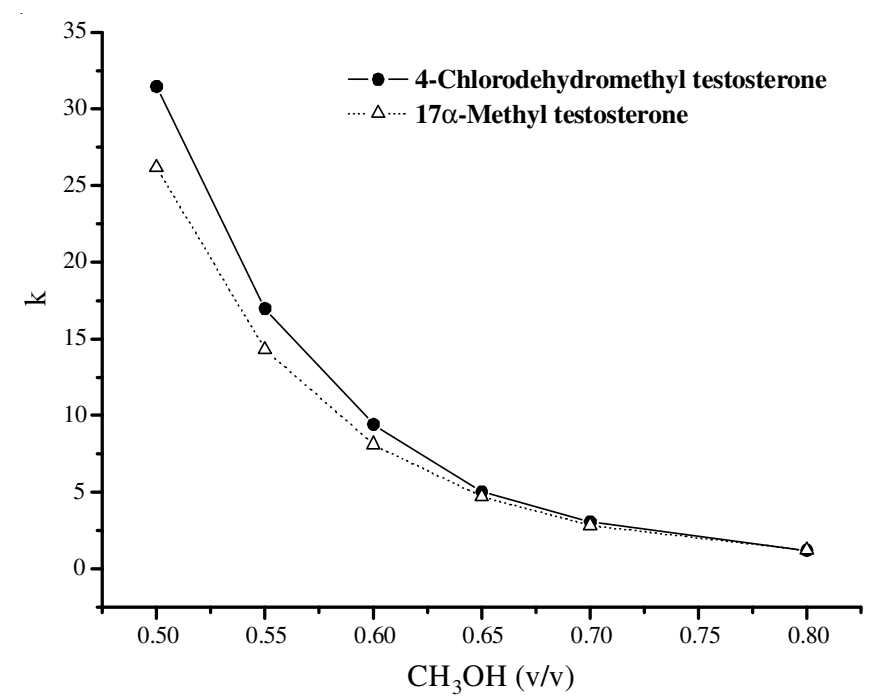

Fig. 4. Influence of methanol proportion in mobile phase on retention of methyl testosterone and 4-chlorodehydromethyl testosterone. Other HPLC conditions were the same as in Fig. 2

Detection wavelength: For the HPLC analysis, $254 \mathrm{~nm}$ was considered as the detection wavelength because it is close to the maximum absorption of both methyl testosterone and 4-chlorodehydromethyl testosterone (Fig. 3). In addition, it is commonly used in routine analysis, especially in the laboratories without a tunable or DAD UV-VIS absorbance detector.
Electrospray ionization mass spectrum: It is found that the sequence of the peaks of methyl testosterone (-11 $\mathrm{min})$ and 4-chlorodehydromethyl testosterone (-10 min) was reversed (Fig. 5) when the analytes were eluted on an Agilent Zorbax $\mathrm{SB}-\mathrm{C}_{18}$ column by the mobile phase composed of methanol and water $(75: 25, \mathrm{v} / \mathrm{v})$. Quasi-molecular ions of methyl testosterone and 4-chlorodehydromethyl testosterone were detected by full scan MS with the $m / z$ range 50-500. The quasi-molecular ions of methyl testosterone in positive and negative mode respectively, $[\mathrm{M}+\mathrm{Na}]^{+},[\mathrm{M}+\mathrm{H}]^{+}$and $[\mathrm{M}-\mathrm{H}]^{-}$, were observed at $\mathrm{m} / \mathrm{z} 325.4,303.3$ and $\mathrm{m} / \mathrm{z} 301.2$ (Fig. 6a,b), while the quasi-molecular ions for 4-chlorodehydromethyl testosterone, $[\mathrm{M}+\mathrm{Na}]^{+},[\mathrm{M}+\mathrm{H}]^{+}$and $[\mathrm{M}-\mathrm{H}]^{-}$, were observed at $\mathrm{m} / \mathrm{z} 357.3, \mathrm{~m} / \mathrm{z} 335.3$ and $\mathrm{m} / \mathrm{z} 333$.3. In addition, the fragment ion of 4-chlorodehydromethyl testosterone, $[\mathrm{M}-\mathrm{OH}]^{+}$was observed at $m / z 317.3$ (Fig. 6c,d). Thus, the MS information indicated that relative molecular weight of methyl testosterone and 4-chlorodehydromethyl testosterone were 302 and 334, respectively. Then $\mathrm{MS}^{2}$ spectra were measured to further confirm the structures of methyl testosterone and 4-chlorodehydromethyl testosterone (Figures not shown). For methyl testosterone, $[\mathrm{M}-\mathrm{H}]^{-}(\mathrm{m} / \mathrm{z}, 301.2)$ was selected as the precursor ion because this ion in full scan MS was the most abundant one (Fig. 6b). The parent ion at $\mathrm{m} / \mathrm{z} 301.2$ exhibited main fragments with $\mathrm{m} / \mathrm{z}$ of 284 and $\mathrm{m} / \mathrm{z} 270$. The fragment of $\mathrm{m} / \mathrm{z} 284$ was the product ion of this precursor ion undergoing a loss of hydroxyl group $\left([\mathrm{M}-\mathrm{H}-\mathrm{OH}]^{-}\right)$. The fragment ion of $m / z 270$ came from the further loss of methyl group ([M-H-OH- $\left.\mathrm{CH}_{3}\right]^{-}$) out of the fragment ion at $m / z$ 284.0. Similarly, the most abundant ion at $\mathrm{m} / \mathrm{z} 333.3$ in Fig. 6d was selected as the precursor ion to obtain the $\mathrm{MS}^{2}$ of 4-chlorodehydromethyl testosterone. The fragments of $\mathrm{m} / \mathrm{z} 318.0$ and $\mathrm{m} / \mathrm{z} 298.0$ were the product ions of this precursor ion undergoing the loss of a methyl group $\left(\left[\mathrm{M}-\mathrm{CH}_{3}\right]^{-}\right)$ and a chlorine atom $\left([\mathrm{M}-\mathrm{Cl}]^{-}\right)$, respectively. Till now, reasonable explanations have been given for each quasi-molecular ions and predominant fragment ions, which were in full agreement with structural characteristics of methyl testosterone and 4chlorodehydromethyl testosterone, respectively and offered an important criterion for identification of these two steroids by LC-ESI-MS.

Application of the method to potentially counterfeit 4-chlorodehydromethyl testosterone tablet: The performance 


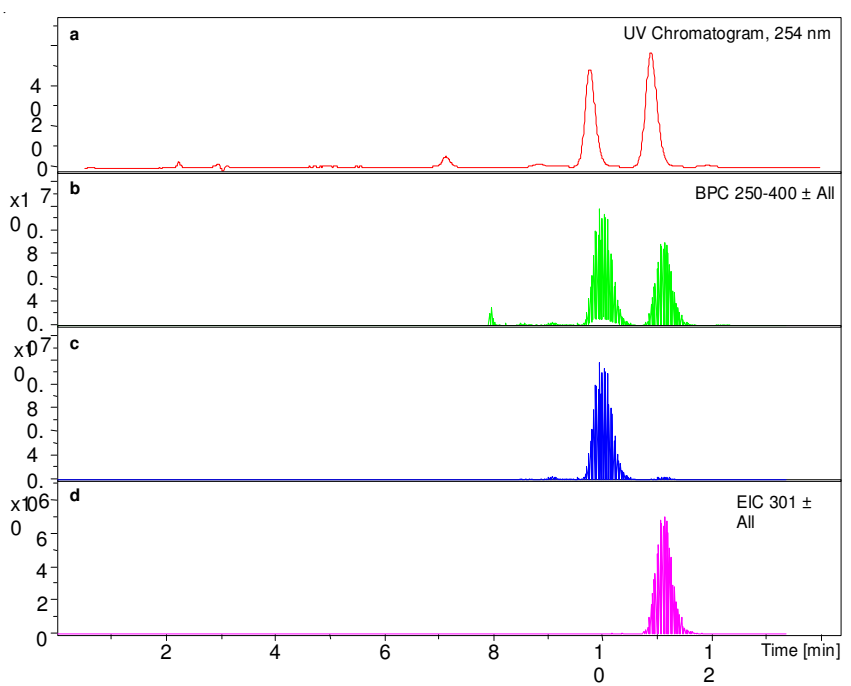

Fig. 5. UV-VIS chromatogram (a) and total ion chromatogram (TIC) in full scan mode (b) of compound 4-chlorodehydromethyl testosterone tablet solution, and selected ion chromatograms (SIMs) of CDM (c) and methyl testosterone (d). Instrumentation: Agilent technologies 1100 series LC/MSD Trap SL system. Column: Agilent Zorbax SB- $\mathrm{C}_{18}$ column $(5 \mu \mathrm{m}, 250 \times 4.6 \mathrm{~mm}$ i.d. $)$. Column temperature: 30 ${ }^{\circ} \mathrm{C}$. Mobile phase: methanol-water $(75: 25$, v/v). Flow rate: $1.0 \mathrm{~mL} /$ min. Injection volume: $10 \mu \mathrm{L}$. UV-VIS detection: $254 \mathrm{~nm}$ at DAD (a). MS detection: capillary voltage: $3500 \mathrm{~V}$; dry temperature: 325 ${ }^{\circ} \mathrm{C}$; dry gas flow rate: $5.00 \mathrm{~L} / \mathrm{min}$; nebulizer pressure: 5 psi; dwell time: $300 \mathrm{~ms}$; TIC under ESI ( \pm ), scanning mass range: 50-500 amu, only the partial MS from 250-400 amu was given (b); SIM under ESI (-) at $m / z 333$ amu (c) and 301 amu (d). Peaks: 1. methyl testosterone; 2. 4-chlorodehydromethylte-stosterone
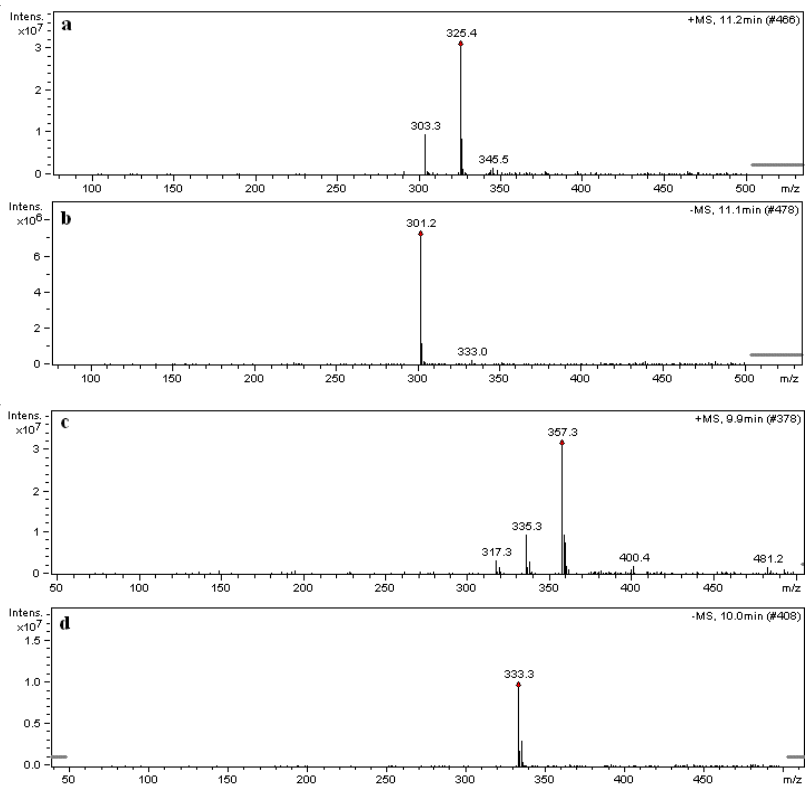

Fig. 6. Mass spectra of methyl testosterone $(\mathrm{a}, \mathrm{b})$ and 4-chlorodehydromethyl testosterone $(\mathrm{c}, \mathrm{d})$ in compound 4-chlorodehydromethyl testosterone tablet solution. a,c. ESI (+); b,d. ESI (-). Collision energy: $0.8 \mathrm{eV}$. Other LC-ESI-MS conditions were the same as in Fig. 5

of the method has been discussed above and then applied to the analysis of steroid 4-chlorodehydromethyl testosterone tablets obtained from Chinese market. Only approximate $7 \%$ of declared active ingredient 4-chlorodehydromethyl testosterone was found (declared content was $-20 \%$ ). Moreover, a high quantity of methyl testosterone was observed as well, which means 4-chlorodehydromethyl testosterone was partly substituted by cheaper methyl testosterone in this counterfeit 4-chlorodehydromethyl testosterone tablet which is labeled to contain 4-chlorodehydromethyl testosterone only.

\section{Conclusion}

A chromatographic method was developed to baseline separate methyl testosterone and 4-chlorodehydromethyl testosterone. The separation was achieved using isocratic reversed-phase chromatography on a $\mathrm{C}_{18}$ column with good linearity, precision and accuracy. The potential counterfeit methyl testosterone can be distinguished by its retention time, UV spectra and $m / z 284$ and 270 when performing LC-MS/ MS. The method was applied successfully to check substitutes of the more expensive active ingredient 4-chlorodehydromethyl testosterone with the cheaper counterfeit methyl testosterone in commercial 4-chlorodehydrome-thyltestosterone tablet. In addition, the fragmentation pattern for these two steroids by LC-ESI-MS/MS was elucidated for the first time, in spite the EI-MS of methyl testosterone has been reported elsewhere.

\section{ACKNOWLEDGEMENTS}

This work was supported by National Basic Research Program of China (973 program, 2009CB421601, 2011CB911003), National Natural Science Foundation of China (90913012), National Science Funds for Creative Research Groups (20821063) and the Analysis \& Test Fund of Nanjing University.

\section{REFERENCES}

1. World Health Organization. Medicines: Counterfeit Medicines. http:// www.who.int/mediacentre/factsheets/fs275/en/index.html (Accessed Jan., 2010).

2. K. Ahmad, Lancet, 363, 713 (2004)

3. World Health Organization, Counterfeit Drugs. Guidelines for the Development of Measures to Combat Counterfeit Drugs, WHO/EDM/QSM/ 99.1, WHO (1999).

4. H.Y. Li, Q.M. Zhang, X.D. Li and J.M. Wu, J. Pharm., 20, 195 (2005).

5. Wikipedia. http://en.wikipedia.org/wiki/Methyl testosterone (Aug. 2nd, 2011).

6. Wikipedia. http://en.wikipedia.org/wiki/4-Chlorodehydromethyl testosterone (Feb. 27th, 2011).

7. M.D. Green, D.L. Mount, R.A. Wirtz and N.J.A. White, J. Pharm. Biomed. Anal., 24, 65 (2000).

8. K.A. Hall, P.N. Newton, M.D. Green, M. De Veij, P. Vandenabeele, D. Pizzanelli, M. Mayxay, A. Dondorp and F.M. Fernandez, Am. J. Trop. Med. Hyg., 75, 804 (2006).

9. C. Eckers, N. Haskins and J. Langridge, Rapid Commun. Mass Spectrom., 11, 1916 (1997).

10. N.J. Haskins, J. Pharm. Biomed. Anal., 25, 767 (2001).

11. The United States Pharmacopeia and the National Formulary, United States Pharmacopoeia Convention, Rochville, USA (2007).

12. X.M. Ouyang, S.L. Ren and Y.M. He, Chin. J. Hosp. Pharm., 21, 651 (2000).

13. R. Gonzalo-Lumbrersa and R. Izquierdo-hornillos, J. Pharm. Biomed. Anal., 32, 433 (2003).

14. S.M.R. Stanley, L. Smith and J.P. Rodger, J. Chromatogr. B, 690, 55 (1997).

15. A.A.M Stolker, L.A van Ginkel, R.W Stephany, R.J Maxwell, O.W Parks and A.R Lightfield, J. Chromatogr. B, 726, 121 (1999).

16. F. Buiarelli, G.P. Cartoni, L. Amendola and F. Botrè, Anal. Chim. Acta, 447, 75 (2001).

17. National Institute of Standards and Technology. NIST Chemistry WebBook, http://webbook.nist.gov/cgi/cbook.cgi?ID=C58184\& Units=SI\&Mask =200\#Mass (2011). 